\title{
Mimulus is an emerging model system for the integration of ecological and genomic studies
}

\author{
CA Wu, DB Lowry, AM Cooley, KM Wright, YW Lee and JH Willis \\ Department of Biology, Duke University, Durham, NC, USA
}

The plant genus Mimulus is rapidly emerging as a model system for studies of evolutionary and ecological functional genomics. Mimulus contains a wide array of phenotypic, ecological and genomic diversity. Numerous studies have proven the experimental tractability of Mimulus in laboratory and field studies. Genomic resources currently under development are making Mimulus an excellent system for determining the genetic and genomic basis of adaptation and speciation. Here, we introduce some of the phenotypic and genetic diversity in the genus Mimulus and highlight how direct genetic studies with Mimulus can address a wide spectrum of ecological and evolutionary questions. In addition, we present the genomic resources currently available for Mimulus and discuss future directions for research. The integration of ecology and genetics with bioinformatics and genome technology offers great promise for exploring the mechanistic basis of adaptive evolution and the genetics of speciation.

Heredity (2008) 100, 220-230; doi:10.1038/sj.hdy.6801018; published online 6 June 2007

Keywords: adaptation; ecological genetics; floral evolution; Mimulus guttatus; Mimulus lewisii; speciation

The broad goal of ecological and evolutionary functional genomics (EEFG) is to understand both the evolutionary processes that create and maintain genomic and phenotypic diversity within and among natural populations and species, and the functional significance of such variation. For the first time, the tools of genomics allow researchers to address in a direct and meaningful way fundamental questions about adaptive evolution and the origin of biological diversity in natural settings. Historically, progress towards a mechanistic understanding of the genetics of adaptation and speciation has been slow. Technical limitations have restricted study to a small number of genes in a handful of model organisms. However, recent advances in our ability to characterize whole genomes and evaluate the functional significance of individual genes are rapidly changing the intellectual landscape. Furthermore, because the genomic tools and information developed in model systems can be increasingly extended to non-model species that are otherwise well suited for answering evolutionary questions, the challenge of understanding the molecular basis of evolution of natural populations and species can now be addressed.

The process of creating a new model system particularly suitable for such synthetic EEFG studies is not trivial. Ideally such a species, or group of closely related species, should have attributes that allow for direct genetic and molecular analysis of important phenotypic variation. Clearly, it is important that the system be rich in ecologically and evolutionarily interesting traits, and that these traits vary between interfertile individuals.

Correspondence: Dr JH Willis, Department of Biology, Duke University, Box 90338, Durham, NC 27708-0338, USA.

E-mail: jwillis@duke.edu

Received 8 September 2006; revised 22 December 2006; accepted 19

April 2007; published online 6 June 2007
Because the expression of such fitness traits can vary depending on the environment (for example, Campbell and Waser, 2001), a comprehensive assessment of the adaptive significance of these traits also requires the ability to conduct experiments such as reciprocal transplants under natural field conditions. Incomplete reproductive isolation between important variants or species is necessary for genetic mapping, which can serve as a first step towards defining the genetic, and ultimately the molecular, basis of focal traits. Logistically, conducting such genetic studies requires a system with a reasonably short generation time, high fecundity and amenability to laboratory or greenhouse culture.

Finally, for emerging model systems, substantial effort is required to develop the comprehensive genomic resources needed to investigate the molecular genetic basis of adaptation and speciation. Numerous highly polymorphic molecular markers are needed for genetic mapping of the genomic regions responsible for variation in ecologically interesting traits. If the genetic markers used for linkage mapping are also located on an ordered physical map of genomic clones, then it is possible to go from Quantitative Trait Locus (QTL) mapping of traits and subsequent fine-mapping to positional cloning and functional analysis of individual genes contributing to the phenotypic variation. To date, we are unaware of non-crop plant systems outside of Arabidopsis in which the genetic basis of natural phenotypic variation has been successfully identified using such an approach. The ultimate physical map, of course, is the entire sequenced genome, which if properly annotated with gene structure and functional information, can help identify candidate genes in genomic regions discovered through QTL mapping.

The wildflower genus Mimulus (Phrymaceae ex. Scrophulariaceae) is one such new model system that is 
being used to elucidate the genetic mechanisms of adaptation and speciation in an ecological context. Members of the genus vary in numerous ecologically relevant features. Genetic diversity is high, and interspecific crossing barriers range from complete to virtually nonexistent. Mimulus species also have many attributes that facilitate genetic experimentation. All species in the genus are self-compatible, many can be clonally propagated from cuttings, and most have only a 6-12 week generation time under experimental conditions. Mimulus flowers are easily emasculated and hand pollinated, and most species produce hundreds of seeds in a single cross. Mature plants are sufficiently small that they can easily be grown in large numbers in small growth chambers. Until recently, however, the relative lack of molecular genetic resources severely restricted the potential for functional genomic studies of adaptation and speciation in these plants.

In the past 2-3 years, a team of researchers, with support of the National Science Foundation, has made substantial progress in developing genomic resources in each of two distantly related groups of species, the Mimulus guttatus and the Mimulus cardinalis species complexes. These resources now include 15000 raw expressed sequence tags (ESTs) developed from flower bud tissue, almost 1000 highly polymorphic gene-based markers suitable for use in a broad array of Mimulus species, detailed linkage maps, BAC libraries for $M$. guttatus and $M$. lewisii (selected as a representative of the $M$. cardinalis complex), integrated genetic and physical maps, seed stocks and a centralized web site (http:// www.mimulusevolution.org), which makes these data and resources available to the entire scientific community. Because of its rich history of ecological and evolutionary research, amenability to genetic experimentation and rapidly developing genomic resources,
M. guttatus was selected by Department of Energy's Joint Genome Institute (JGI; http:/ / www.jgi.doe.gov) for whole-genome sequencing. JGI initiated this sequencing project in 2006, and has already completed over $70 \%$ of the sequencing for the $430 \mathrm{Mbp}$ genome. As sequencing progresses, the raw sequences are immediately made available to the public through National Center for Biotechnology Information's Trace Archive (http:// www.ncbi.nlm.nih.gov/Traces). An additional 200000 M. guttatus ESTs and $20000 \mathrm{M}$. lewisii ESTs are also being sequenced by JGI. A complete genomics toolkit for Mimulus - including biological, genetic and bioinformatic resources - will now allow researchers to address the real challenge of understanding how genomic complexity and diversity evolve in natural populations and species (Figure 1).

\section{Diversity within Mimulus}

Mimulus has been the subject of intensive ecological and evolutionary genetic research for over 50 years. Species within Mimulus have become key systems for investigating the genetics of speciation (Hiesey et al., 1971; Bradshaw et al., 1998; Sweigart et al., 2006), inbreeding depression (Dudash and Carr, 1998), mating system evolution (Leclerc-Potvin and Ritland, 1994; Fishman et al., 2002; Sweigart and Willis, 2003), ecological adaptations (Macnair, 1983; Angert and Schemske, 2005) and cytological patterns of evolution (Vickery, 1978; Beardsley et al., 2004). In addition to being attractive for comparative studies within the genus, Mimulus is also phylogenetically well placed for broad comparative genomics research across the plant kingdom, based on its relatedness to other model systems for floral development (Antirrhinum; also Lamiales), to crop plants with well-developed genomic resources (for

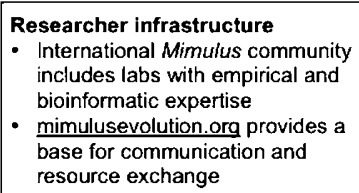

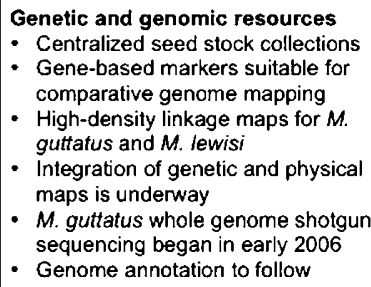

Evolutionary relevance
- Documented genetic variation in
natural populations
QTL mapping studies to identify
genetic regions affecting fitness
components
- Phylogenetic history of Mimulus
enables inference of trait evolution
among groups
- Amenable to controlled field
experiments

Ecological context

Species are widely distributed

both geographically and edaphically

Many populations or species are habitat-specialists

Field populations are located on private and federally protected lands

private and federally protected
lands

Figure 1 Network of resources available for the Mimulus community of researchers. The monkeyflower genus Mimulus possesses a wide array of biological characteristics and developing tools that make it an emerging model for ecological and evolutionary functional genomics. Figure based on criteria proposed by Feder and Mitchell-Olds (2003). 
example, Lycopersicon, Helianthus, Lactuca; all Asterids) and to Arabidopsis (both Core Eudicots).

Mimulus occurs worldwide, with the majority of the 160-200 species belonging to two large radiations that are centered in Western North America and Australia (Vickery, 1978; Beardsley and Olmstead, 2002; Beardsley et al., 2004). Rapid adaptive divergence appears to be a characteristic of the group; most of the taxonomic sections contain groups of closely related species that are interfertile, yet remain ecologically and morphologically distinct (Vickery, 1978). The genus as a whole contains tremendous phenotypic variation, which is mirrored within each species group and even within some species. Mimulus occupies habitats from desert to aquatic to alpine, includes annual, herbaceous perennial and woody perennial growth forms, and contains mating systems ranging from completely outcrossing to obligately selfing to obligately asexual (Grant, 1924; Vickery, 1978; Beardsley et al., 2004).

Much of this diversity is represented within two groups of species that contain the close relatives of M. guttatus and M. lewisii (Table 1). The yellow monkeyflowers of the $M$. guttatus species complex show remarkable variation in mating system, life history and edaphic range, which has made the group a premier system for investigating the genetics of adaptive divergence and speciation in plants (for example, Kiang and Hamrick, 1978; Macnair and Cumbes, 1989; Fenster and Ritland, 1994; Fishman et al., 2002; Hall and Willis, 2005). With the exception of a few South American species, the ranges of most members of the $M$. guttatus complex are sympatric with that of $M$. guttatus. The low genetic distances among taxa indicate that this clade is relatively young, with very closely related constituent species (Beardsley et al., 2004). These taxa vary in the amount and patterns of molecular genetic varation in a manner consistent with their mating systems. Population surveys of microsatellite variation (Awadalla and Ritland, 1997; Kelly and Willis, 1998) and nucleotide polymorphism (Sweigart and Willis, 2003) show that the predominately outcrossing $M$. guttatus contains much more genetic diversity at the species level and within populations than its self-fertilizing relatives like $M$. nasutus. Indeed, estimates of nucleotide diversity in M. guttatus at the two nuclear genes studied to date are substantially higher than most well-studied plant and animal species (Sweigart and Willis, 2003).

The herbaceous perennials that comprise the $M$. cardinalis species complex, including $M$. lewisii, have long served as models for the study of ecological adaptations, as closely related species typically show distinct geographic ranges (Beardsley et al., 2003). In particular, the widespread sister species $M$. cardinalis and $M$. lewisii have been the subject of several landmark studies on reproductive isolation stemming from adaptation to pollinators (Schemske and Bradshaw, 1999; Bradshaw and Schemske, 2003) and abiotic conditions (Hiesey et al., 1971; Ramsey et al., 2003; Angert and Schemske, 2005).

Here, we focus primarily on recent progress towards identifying the underlying genetic basis of ecologically and evolutionarily interesting phenotypes in the $M$. guttatus and $M$. cardinalis species complexes. We restrict most of our review to these two species complexes because they have become well-established study sys- tems, with rapidly expanding genomic tools. Ultimately, we expect that these resources will prove applicable to and complement the ongoing research programs in numerous other Mimulus systems. Indeed, multiple gene-based PCR-amplified markers developed for $M$. guttatus have already successfully amplified in several other Mimulus, in both closely (for example, M. tilingii, $M$. cupreus and $M$. luteus) and distantly (M. primuloides, $M$. lewisii, M. aurantiacus and $M$. ringens) related species (unpublished data).

\section{Habitat adaptations}

Mimulus species have evolved a stunning variety of life history, developmental and physiological traits in response to a broad range of habitats. The $M$. guttatus species complex alone contains populations found from sea level to elevations over 10000 feet, in grasslands, forests, desert streams and basalt cliffs, peat bogs, alpine meadows and seeps, serpentine barrens, coastal cliffs and sand dunes, toxic copper mine tailings, and even on the edges of geysers in the Western USA (Figure 2). Ongoing research is examining the genetic basis of many of these adaptations to local environmental conditions. For example, differential adaptations to elevation that limit species ranges have been shown in $M$. lewisii and its sister species $M$. cardinalis through reciprocal transplant experiments (Hiesey et al., 1971; Angert and Schemske, 2005). Mimulus guttatus and related species are well known for the evolution of tolerance to normally toxic soils. Adaptation to copper mine tailings has been documented in many populations of M. guttatus (Allen and Sheppard, 1971; Macnair et al., 1989) as well as in $M$. cupriphilus, a self-fertilizing species only found on abandoned copper mines in and around Copperopolis, CA (Macnair, 1989). The copper tolerance in both species appears to be largely due to the same single Mendelian locus, although the gene(s) causing the tolerance has yet to be identified (Macnair, 1983; Macnair and Cumbes, 1989). Mimulus guttatus and the closely related $M$. nudatus have evolved the ability to thrive on the serpentine soils and outcrops commonly found throughout Western North America, often via tolerance to multiple heavy metals like nickel, as well as to rapid seasonal onset of drought. The genetic basis of these adaptations, however, is yet to be determined (Gardner and Macnair, 2000; Hughes et al., 2001).

Populations of $M$. guttatus and its relatives occupy habitats that vary drastically in the seasonal availability of soil moisture. Adaptation to this heterogeneity of moisture availability has been correlated with divergence in mating system, flowering time, morphology and life history (Kiang and Hamrick, 1978). However, clear experimental demonstration of the adaptive significance of these traits has until recently been inconclusive.

Research on coast and inland populations of $M$. guttatus has led to a better understanding of the genetic basis of geographic race formation, and the potential role of water availability in this process. Inland populations of $M$. guttatus are found frequently across Western North America as diminutive annual herbs that flower in early spring, whereas a late-flowering large perennial ecomorph (M. guttatus ssp. grandis Greene) is found exclusively in continually moist coastal habitats. Hall et al. (2006) conducted QTL analyses in an F2 mapping 
Table 1 Phenotypic diversity in two Mimulus species complexes

Species Notable characteristics

Mimulus guttatus complex members

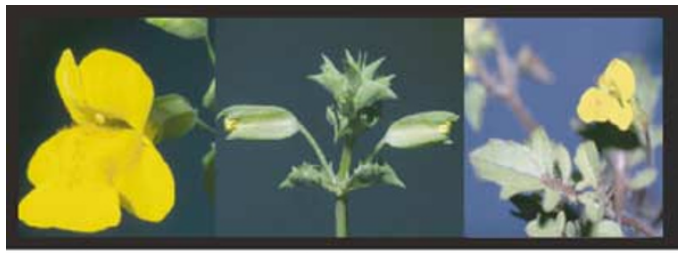

a b c

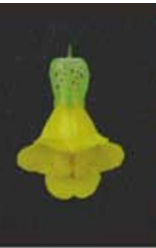

d

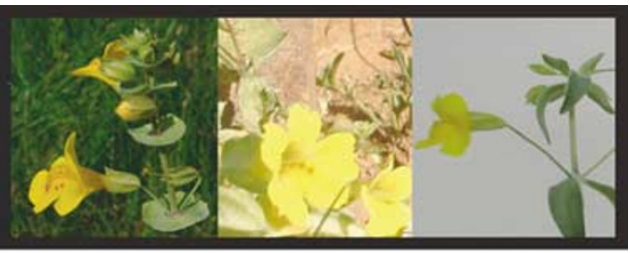

e

f

g
(a) M. guttatus
(b) M. nasutus
(c) M. laciniatus
(d) M. platycalyx
(e) M. glaucescens
(f) M. cupriphilus
(g) M. nudatus

Most common species in the M. guttatus complex; geographically widespread and highly polytypic Highly selfing mating system, with either open or closed, cleistogamous flowers

Distinguished by its highly dissected leaves; restricted to sites $>900 \mathrm{~m}$ in the California Sierra Nevada; selfing

Showy-flowered, yet highly selfing, with cup-shaped calyx

Restricted to the foothills of the Cascade Range and Sierra Nevada in central California, with fused floral bracts

Endemic to copper mines in central California, selfing mating system; distinct 'tooth' on petals

Endemic to serpentine soils, with tiny linear leaves

Other closely related species complexes

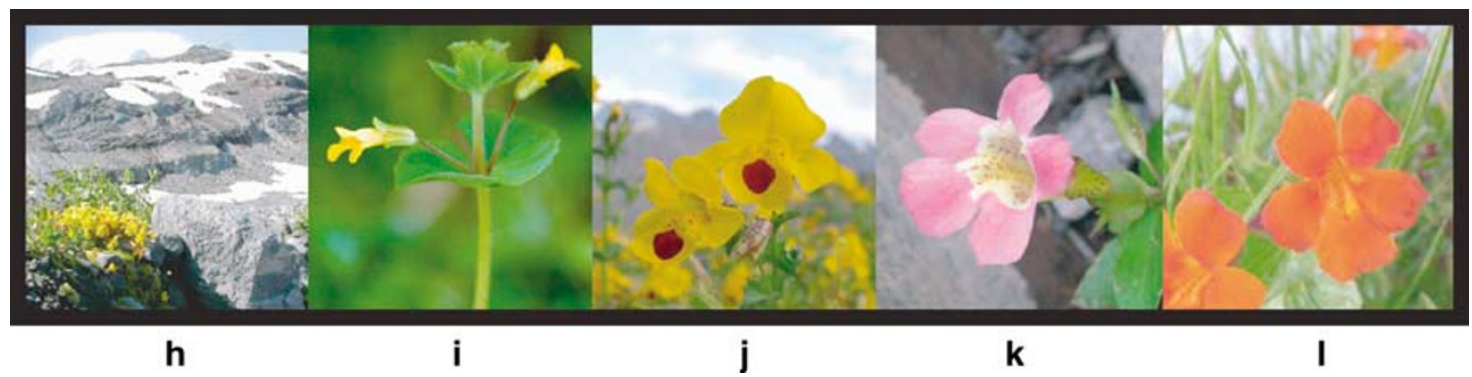

(h) M. tilingii complex

(i) M. glabratus complex

$(\mathrm{j}, \mathrm{k}, \mathrm{l})$ M. luteus complex

Restricted high montane and alpine distribution; often in dense clusters among moss in rocky mountain streams

Widespread group with a north-to-south geographic series of ploidy, $n=15,30,31,46$ from Canada through South America. Taxa vary in breeding system

Closely related taxa that co-occur in the Andes and vary in floral pigment patterning

Mimulus cardinalis complex members

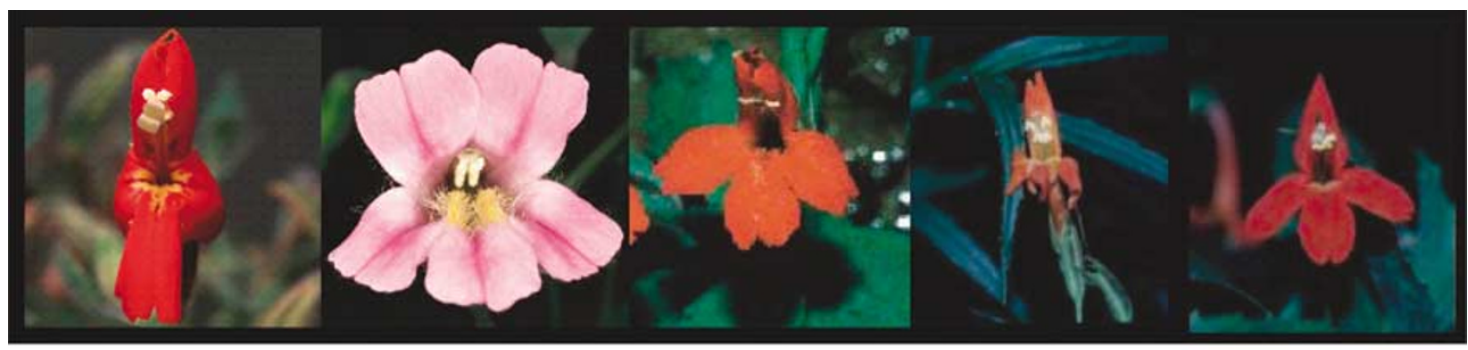

m

(m) M. cardinalis

(n) M. lewisii

(o) M. eastwoodiae

(p) M. nelsonii

(q) M. verbenaceus $M$. rupestris (not shown) n

o p

The M. guttatus and M. cardinalis species complexes described by Vickery (1978) each contain numerous ecologically and morphologically distinct but interfertile species that are well suited for studying ecological and evolutionary genetics in nature. Recent genetic analyses (Beardsley et al., 2004) indicate phylogentic support for including the M. tilingii, M. glabratus and M. luteus complexes in the M. guttatus complex. Photographs reproduced with permission from George Hartwell (e), Charles Peirce (i), and Paul Beardsley (o, p, q). 

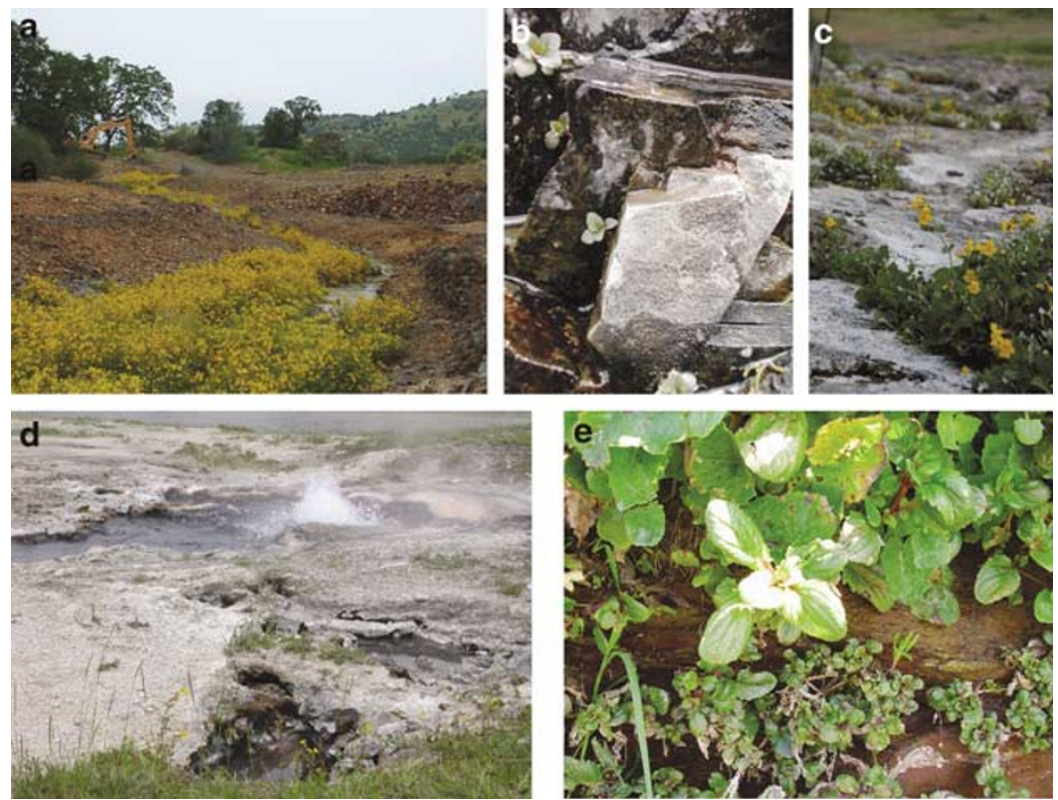

Figure 2 Extreme habitats occupied by Mimulus guttatus. (a) Copper mine tailings. (b) Rocky montane outcrops. (c) Serpentine seeps. (d) Edges of geysers and hotsprings. (e) The spash zone of the Pacific Ocean. Photograph $2 \mathrm{~d}$ is reproduced, with permission, from L Fishman.

population from a cross between such coast and inland plants. Two pleiotropic QTLs of large effect were found to each contribute $20-30 \%$ of divergence in multiple morphological and life-history traits between the ecomorphs, along with many additional loci of minor effect. A reciprocal transplant experiment with recombinant inbred lines subsequently demonstrated strong selection for early flowering in the inland habitat and selection for later flowering at the coast. The role of water availability in this process seems clear: slow developing plants do not survive to flower at the inland site because they cannot tolerate the summer drought (Hall and Willis, 2006).

The divergence between ecogeographic races such as these has long been the focus of evolutionary biologists (Turesson, 1922; Stebbins, 1950). Clausen (1951) suggested such geographic races are reproductively isolated, and therefore constitute incipient species. The genetic resources available in $M$. guttatus are now being used to test this proposal, by determining whether gene flow is restricted between the coast and inland populations through population structure analyses. Further, the combination of fine-mapping approaches and genomic tools will facilitate determination of the genetic basis of ecological reproductive isolation between these two divergent races.

This broad ecological diversity in Mimulus makes it an excellent system to examine the genetic basis of adaptation to ecological heterogeneity. As QTLs are identified for particular adaptive traits, it will be possible to determine whether the same or different genetic architecture is involved in parallel adaptations, and whether new mutations or standing genetic variation underlies shifts to new habitat (Colosimo et al., 2005). Studies of the genetic basis of adaptation in Mimulus will provide a more comprehensive understanding of the formation of edaphic endemics and ecogeographic races. Such research with $M$. guttatus will help to determine the geographic mode and genetic basis of ecological speciation.

\section{Floral evolution}

The evolution of floral form has long been a major focus of evolution and ecology, with pollinator-mediated selection often considered the critical factor in the evolution of floral diversity (Grant and Grant, 1968; Stebbins, 1970). Although predominantly composed of bee-pollinated species, Mimulus also includes members that are pollinated by hummingbirds, hawkmoths, bombyliids and other flies. Multiple pollinator shifts are evident among Mimulus species, including at least three independent recent origins of hummingbird pollination (Beardsley and Olmstead, 2002). In addition, beepollinated Mimulus species have been found to vary in pollinator guild structure across different geographic regions (Waser et al., 1982). The diversity of Mimulus pollinators, combined with the frequent evolution of selffertilization in Mimulus (Vickery, 1978; Ritland and Ritland, 1989; Fishman et al., 2002; Beardsley et al., 2003; Whittall et al., 2006), has likely contributed to the vast diversity of floral form in the genus.

Recent work on floral evolution demonstrates that fundamental evolutionary questions can be addressed in Mimulus through the combination of field experiments and modern genomic approaches. Bradshaw et al. (1995, 1998) pioneered the application of genome mapping to study of ecologically important traits in Mimulus using RAPD and allozyme markers to map floral QTLs underlying the divergence between red-flowered, hummingbird-pollinated $M$. cardinalis and pink-flowered, bee-pollinated $M$. lewisii. The initial mapping experiments, with hybrid phenotypes measured in controlled greenhouse environments, revealed QTLs with major effects on virtually every floral character studied, from coloration and morphology to nectar production. To 
determine the effect of these QTLs on pollinator visitation and discrimination, Schemske and Bradshaw (1999) moved the genotyped hybrids to a field site near one of the few regions where the species coexist, and observed bee and hummingbird visitation behavior. Amazingly, the $M$. cardinalis allele at a single QTL, YELLOW UPPER (YUP), was responsible for an $80 \%$ loss of visitation by bee pollinators, and the $M$. cardinalis allele at a QTL responsible for variation in nectar production doubled hummingbird visitation (Schemske and Bradshaw, 1999). Bradshaw and Schemske (2003) subsequently created near-isogenic lines (NILs), where heterospecific alleles at YUP were reciprocally introgressed into the parental genetic backgrounds, and evaluated the response of pollinators to the NILs in the field. They observed an even clearer pattern of pollinator discrimination due to this locus, with a 74-fold increase in bee visitation in $M$. cardinalis NILs that carried the $M$. lewisii YUP allele, and a 68-fold increase in hummingbird visitation in $M$. lewsii NILs with the M. cardinalis YUP allele. Although the ecological context, in this case the community of potential pollinators, is certainly important to the evolution of new pollinator associations, these results also demonstrate that single genomic regions can have a large effect on major evolutionary transitions.

The recently expanded genomic resources for the $M$. cardinalis species complex will soon make it possible to more precisely map and clone the major genes underlying floral evolution in the $M$. cardinalis/M. lewisii system. As the genes involved in hummingbird pollination are identified in $M$. cardinalis, it will be interesting to determine whether homologous genetic mechanisms are involved in the parallel evolution of hummingbird pollination in other Mimulus lineages (Beardsley et al., 2003).

In contrast to evolution of hummingbird pollination, many genes of small effect seem to be involved in floral transitions associated with the evolution of self-pollination. Classic biometrical studies involving large-flowered, outcrossing M. guttatus and several derived small flowered selfing species provided the initial evidence in support of this view (Macnair and Cumbes, 1989; Fenster et al., 1995). In the first QTL mapping experiment in the M. guttatus species complex, Lin and Ritland (1997) identified several minor floral loci. Owing to incomplete marker coverage of the genome and a somewhat small mapping population, they concluded that while they were unable to detect additional QTLs of small effect, most of the floral differences between $M$. guttatus and $M$. platycalyx likely were highly polygenic. More recently, Fishman et al. (2002) were able to identify more than 20 loci of small to moderate effects underlying species differences in floral morphology related to mating system divergence between the highly self-fertilizing $M$. nasutus and $M$. guttatus, using a large mapping population and numerous informative markers that nearly covered the complete genome. Most of those floral QTLs appeared to influence variation in several aspects of floral size and shape, a result that suggests the importance of pleiotropy in the evolution of complex morphologies. Ongoing fine-scale mapping will determine if these are indeed pleiotropic QTLs, or instead are tightly linked genes with individual effects.

To what extent does the genetics of species differences mirror the genetics of variation within species? The polygenic, pleiotropic genetic architecture of floral morphological differences between selfing and outcrossing species also appears to be characteristic of floral variation among divergent populations of $M$. guttatus. It may also be characteristic of standing variation for floral traits within populations. Hall et al. (2006) identified numerous pleiotropic, small-effect QTLs contributing to the substantial divergence in flower size between alpine annual and coastal perennial forms of $M$. guttatus, although they also found two QTLs that each contributed to $20-30 \%$ of the difference between the parents. Genetic mapping studies of floral variation within $M$. guttatus populations are not yet complete, but already it is clear that substantial genetic variation in floral traits exists in all populations studied (Fenster and Ritland, 1994; Robertson et al., 1994; Kelly and Willis, 2001; Kelly, 2003). Is this standing variation merely the result of deleterious mutations with pleiotropic effects on floral morphology, which might not contribute to divergence, or are the alleles at floral QTLs at intermediate frequency, perhaps because of ecologically mediated balancing selection? Biometric tests of these alternatives seem to suggest the latter possibility (Kelly and Willis, 2001; Kelly, 2003). If true, the floral variation in the M. guttatus species complex fits with Darwin's (1859) idea that standing, individual variation within populations serves as the raw material for population and species divergence.

The contrast between these mapping results for floral traits in the M. guttatus complex and those underlying pollinator shifts in the $M$. cardinalis complex suggests that different types of selective pressures can lead to contrasting genetic architectures underlying adaptations. Morphological evolution in which adaptive intermediate phenotypes do not exist, such as floral morphologies that are intermediate to typical pollinator syndromes, are more likely to occur through few loci of large effect (Bleiweiss, 2001). In contrast, morphological evolution in which even small directional changes may be advantageous, such as during a shift from complete outcrossing towards self-fertilization, can occur through many loci of small effect (Fishman et al., 2002).

\section{Reproductive isolation and hybrid incompatibilities}

Reproductive isolation between species or divergent populations typically involves multiple barriers to gene exchange that act either to prevent hybridization or to limit the ability of hybrids to facilitate introgression (Coyne and Orr, 2004). In Mimulus, many of the adaptations to different habitats, pollinators and mating system discussed above may serve as prezygotic isolating barriers between taxa (Schemske and Bradshaw, 1999; Bradshaw and Schemske, 2003; Ramsey et al., 2003). For example, adaptation to different elevational habitats and pollinators are the primary isolating barriers between $M$. lewisii and $M$. cardinalis (Ramsey et al., 2003), whereas adaptive divergence in flowering time, mating system and pollen-pistil interactions cause most of the isolation between $M$. guttatus and $M$. nasutus (Kiang and Hamrick, 1978; Diaz and Macnair, 1999; Martin and Willis, 2007). Such ecologically important traits may also serve as extrinsic postzygotic isolating 
barriers to the extent that hybrids are produced, but exhibit traits that are maladapted to the parental environments (Coyne and Orr, 2004). Although divergence driven by adaptation to ecological conditions is likely the major cause of reproductive isolation in Mimulus, intrinsic postzygotic incompatibilities such as hybrid lethality and sterility may also contribute to species barriers.

Because hybrid sterility or lethality clearly cannot be directly adaptive, a major challenge to evolutionary biologists is to understand how and why incompatibilities tend to accumulate as populations or species diverge. Any attempt at determining whether incompatibilities arise as an incidental by-product of adaptation, random genetic drift, or some other evolutionary force requires detailed investigation at the genetic and ultimately molecular levels. Postzygotic hybrid incompatibilities have been studied in Mimulus for over 50 years, beginning with the classic work of RK Vickery that examined crossing barriers between species. Vickery's research in the $M$. guttatus, M. glabratus and $M$. cardinalis complexes revealed two main results (summarized in Vickery, 1978). First, crossing barriers, such as reduced production of F1 seed, hybrid lethality or sterility, were typically observed in interspecific hybridizations, but were often incomplete. Second, there was considerable variation among populations within species in the degree of postzygotic isolation revealed upon crossing. In fact, depending on the parental populations being studied, the magnitude of postzygotic isolation in interspecific crosses could be either greater or less than that in crosses between geographically dispersed populations of the same species. In some cases, hybrid incompatibilities were associated with obvious changes in ploidy level or chromosome structure (Vickery, 1978), although in most cases the genetic basis of these crossing barriers was not able to be resolved. Nonetheless, that barriers typically only partially reduce hybrid fitness and show substantial variation within species suggested the potential for more detailed and direct genetic analysis of hybrid incompatibilities in a population genetic context.

Macnair and Christie (1983) performed the first direct genetic analysis of hybrid incompatibilities in Mimulus. While studying the genetic basis of copper tolerance in California populations of M. guttatus, they noticed that some crosses between plants from the copper mines and certain other populations resulted in F1s that died as young seedlings. Further crossing studies revealed that the F1 lethality was caused by a deleterious epistatic interaction between the copper tolerance allele from the mine populations (or a gene tightly linked to it) and alleles at an unknown number of different loci from the other populations. Such deleterious interlocus interactions, usually referred to as Dobzhansky-Muller (D-M) incompatibilities, are thought to be the major cause of low hybrid fitness in plants and animals (reviewed in Coyne and Orr, 2004). Remarkably, it appeared that natural selection for copper tolerance had indirectly resulted in the evolutionary origin of the hybrid incompatibility. Christie and Macnair (1984) later identified a second case of hybrid seedling lethality within M. guttatus caused by an even simpler, two-locus D-M interaction. For both of these incompatibility systems, the incompatible alleles were highly restricted in their geographic distribution (Christie and Macnair, 1987).
With the possible exception of the copper tolerance allele, the evolutionary forces acting on these alleles to cause their population distribution remains unknown. However, because these alleles have large effects on hybrid fitness, genetic mapping and cloning of these genes should be feasible, and would elucidate the molecular and developmental basis of the incompatibilities and the copper tolerance. Most importantly, such experiments would also reveal whether the copper tolerance locus is the same gene or merely tightly linked to the hybrid lethal gene, and therefore help determine the role of natural selection in establishing this reproductive barrier.

In Mimulus, partial hybrid sterility is often observed in interspecific crosses (Vickery, 1978). For example, Ramsey et al. (2003) found that F1s from a cross between $M$. lewisii and $M$. cardinalis had almost a $70 \%$ reduction in pollen viability and substantially diminished female fertility. Recently, progress has been made on understanding the genetic basis of hybrid sterility in crosses between $M$. guttatus and $M$. nasutus. Using $M$. nasutus as the maternal parent, Fishman and Willis (2001) observed about a $40 \%$ reduction in pollen viability in F1 and F2 hybrids, with a small proportion of the F2s (but no F1s) being completely male sterile. These results were inconsistent with chromosomal rearrangements causing sterility (where heterokaryotypes should have the lowest fertility), and instead implicated D-M interactions among loci. Further genetic analyses were initially hampered by the lack of sufficient codominant genetic markers for Mimulus, but Sweigart et al. (2006) were able to use recently developed EST markers for these species, in conjuction with complex crossing designs, to map the underlying genetic loci to small genomic regions. This hybrid male sterility was mainly caused by interactions between two unlinked loci, with additional loci having comparatively small effects (Sweigart et al., 2006). The two main loci had asymmetrical effects, as expected with $\mathrm{D}-\mathrm{M}$ incompatibilities, and differed in the degree of dominance: a single copy of the allele at the hybrid male sterility 1 ( $h m s 1$ ) locus from $M$. guttatus caused severe sterility when combined with two alleles from M. nasutus at the hms2 locus, but the reciprocal combinations had high fertility. Interestingly, this genetic incompatibility also caused partial female sterility, as well as a genotypespecific reduction in recombination rates, raising the possibility that the normal function of these genes lies in early stages of male and female gametophyte development, such as meiosis. Further efforts to fine-scale map and clone these two loci are underway.

To investigate the evolutionary history and geographical distribution of these incompatibility alleles, Sweigart et al. (2006) used the test cross approach pioneered in Mimulus by Macnair and Christie (1987), coupled with the use of highly polymorphic genetic markers flanking each locus. The incompatible allele at hms2 appears to be widespread, perhaps fixed throughout the geographic range of $M$. nasutus. Surprisingly, however, the incompatible $h m s 1$ allele is highly restricted to some Oregon Cascades populations of M. guttatus, and appears to be at intermediate frequencies in at least one population. Future field experiments and molecular population genetic studies are needed to better understand the extent to which natural selection, drift and migration shape the distribution of these incompatibility factors. 
D-M incompatibilities are typically described in terms of epistatic interactions among genes in the nuclear genome (reviewed in Coyne and Orr, 2004). However, because nuclear genes often encode proteins that have critical functions in the chloroplast or mitochondrion, it is expected that over time components of the nuclear and cytoplasmic genomes will coevolve. This tight functional integration therefore may be disrupted in hybrids that have heterospecific combinations of nuclear and cytoplasmic genes. Recently, nuclear-cytoplasmic male sterility was discovered in certain $M$. guttatus $\times M$. nasutus hybrids that had inherited their cytoplasmic genomes from the M. guttatus parent (Fishman and Willis, 2006). One-quarter of the F2 hybrids with M. guttatus cytoplasm developed malformed anthers that did not produce pollen grains, but the reciprocal cross did not produce any anther-sterile progeny. The simplest genetic model consistent with these results is that homozygotes for a $M$. nasutus allele at a single nuclear locus are completely male-sterile when they also carry the $M$. guttatus cytoplasm. This was fully supported by genetic mapping experiments based on EST markers that located the nuclear $M$. nasutus component of the incompatibility to single chromosomal region. The M. guttatus parent is therefore fertile, despite having a cytoplasmic genome capable of causing sterility, because the cytoplasmic male sterility (CMS) is masked by a dominant nuclear allele that restores male fertility. While it is possible that this hybrid incompatibility evolved according to the classic D-M scenario, without low fitness during the transitional stages, it is perhaps more likely that the CMS-restorer system evolved through a process of selfish evolution and genomic conflict (Zeh and Zeh, 2005). Clearly, further experimental work is needed to identify the molecular genetic changes at the CMS and restorer loci underlying this form of hybrid sterility, evaluate their ecological fitness effects and examine their biogeographic history.

All of the examples of hybrid incompatibilities discussed above were identified because of obvious phenotypic abnormalities in hybrids, such as pollen sterility or seedling death, but there may be other incompatibilities that produce no visible defects. For example, early death of certain F2 hybrid zygotes may result in only a partial reduction in F1 seed set. Such differential mortality should result in non-Mendelian genotypic ratios (often termed Transmission Ratio Distortion, TRD) at the loci underlying the incompatibility, as well as at tightly linked genetic markers in segregating hybrid populations. Indeed, TRD of markers has been observed in every interspecific genome-wide mapping study conducted to date in Mimulus (Bradshaw et al., 1995, 1998; Lin and Ritland, 1996; Fishman et al., 2001), and has even been observed in crosses between divergent populations of M. guttatus (Hall and Willis, 2005). In some of these studies over $50 \%$ of the markers are significantly distorted, levels far greater than reported in other intraspecific crosses (see Hall and Willis, 2005). The distorted markers tend to be nonrandomly localized to particular chromosomal regions, which may harbor loci that cause zygotic lethality of hybrids, but alternatively they may be loci that experience selection at earlier stages of gamete formation, function or fertilization. Fishman and Willis (2005) used a reciprocal crossing design to investigate the cause of selection acting on the most distorted chromosomal region discovered in $M$. guttatus $\times$ M. nasutus hybrids (Fishman and Willis, 2001). Surprisingly, a single locus causes the virtually complete transmission of the M. guttatus allele, and thus complete distortion against the $M$. nasutus allele, when the F1 serves as the maternal parent, regardless of which parental species it is crossed with. This result rules out hybrid zygote lethality as the cause, but suggests the involvement instead of some form of female meiotic drive or selective postmeiotic ovule or seed abortion. Differential seed or ovule abortion does not seem to be causing the drive, because plants carrying the driving M. guttatus allele have similar numbers of developing seeds as normal plants, compared to the expected $50 \%$ reduction (Fishman and Willis, 2005), leaving open the strong possibility that drive involves female meiosis itself. Such a process would only be effective in outbreeding species such as $M$. guttatus but not $M$. nasutus, which is consistent with the pattern observed. The essentially complete distortion observed in this Mimulus system suggests that the drive takes place during the first meiotic division (Buckler et al., 1999; Fishman and Willis, 2005), and centromeres are particularly attractive candidates to be involved in drive at this stage (Henikoff et al., 2001; Henikoff and Malik, 2002; Malik, 2005). Whether the Mimulus female drive system is an example of rapid centromere divergence resulting from selfish evolution, and whether it contributes to hybrid sterility or other postzygotic incompatibilities, as predicted by models of centromere drive (Henikoff et al., 2001; Henikoff and Malik, 2002), remains to be seen.

\section{Whole-genome duplications}

Recent comparative genomic analysis has revealed that whole-genome duplications are widespread across many taxonomic groups (Wolfe and Shields, 1997; Jaillon et al., 2004). Despite this increased interest in whole-genome duplications, many major hypotheses of polyploidization, especially those involving natural populations, remain untested (Soltis et al., 2003).

Mimulus is a rich system for such studies of genome duplication and chromosomal evolution, although little detailed molecular work has been conducted to date. Beardsley et al. (2004) conclusively documented at least 13 polyploidization events across the genus; however, this is likely an underestimate, as polyploidy has recently occurred multiple times within many species. Independent polyploidization events have been well documented within M. guttatus, as well as in other members of its species complex (Vickery, 1995), although the relative role of auto- versus allopolyploidization is not known. Mimulus glabratus presents an extreme example within the complex, having experienced two very recent rounds of polyploidization, as well as an aneuploidy event between the two polyploidy events (McArthur et al., 1972). Despite its pervasiveness in the M. guttatus complex, however, polyploidy is far less common in other sections of Mimulus, and has not been documented in the M. cardinalis complex (Vickery, 1995).

As such, Mimulus should prove useful in the examination of many major questions regarding whole-genome duplications, particularly following the completion of sequencing M. guttatus. Polyploidy has been proposed to contribute to the adaptation of organisms through an 
immediate increase in genetic variability, as well as the emergence of transgressive characters (Ramsey and Schemske, 2002). Although results from crop studies have generally supported this role of polyploidy in adaptation, Mimulus can be used to explore further this possibility in natural populations. The multiple independent origins of polyploidy within the M. guttatus complex should make it possible to examine whether there are particular rules governing genome remodeling following duplication, such as if certain genes are typically retained or lost. Moreover, Mimulus may be a good system to test whether duplicate gene loss on homologous chromosomes can facilitate the formation of reproductive isolation within a particular polyploidy lineage (Werth and Windham, 2001; Scannell et al., 2006).

\section{Future directions}

Mimulus is rapidly becoming a strong emerging model system for ecological and evolutionary functional genomics. Members of the genus display extraordinary variation in morphology, physiology, mating system and habitat. Closely related species are maintained by numerous reproductive isolating barriers caused by their ecological, developmental and genomic differences. Within species, geographically widespread populations often show local adaptation to a range of both biological and abiotic factors. Over the past several years, considerable progress has been made both in identifying QTLs underlying ecologically important traits, and in developing the genomic resources that will enable dissection of the genes underlying variation in focal traits.

Ultimately achieving this detailed level of understanding - to both identify candidate genes and understand their molecular and ecological significance - will benefit from the continued development of experimental technologies and protocols, and bioinformatic resources. In particular, top priorities include the development of methods for experimental validation of candidate genes, including transformation and RNA interference methodologies. Efficient transformation protocols also will enable the development of T-DNA insertion mutant collections for studies of gene function. The development of microarrays for Mimulus would greatly enhance our ability to understand patterns of gene expression and its contribution to adaptation and speciation, and provide additional tools for high-resolution genetic mapping. There is also an urgent need for genome-wide investigations into patterns of nucleotide diversity within and among Mimulus species. This information will be tremendously useful for investigating the population structure and evolutionary history of species, as well as for evaluating the influence of selective forces and demography on ecologically important candidate genes identified by linkage mapping and positional cloning. Such studies would also provide information on the patterns and levels of linkage disequilibrium (LD) within species. Depending on the scale of LD within and among populations, LD mapping could prove to be a useful method for determining the molecular genetic basis of adaptive traits. Finally, as new, cheaper sequencing technologies become available, it will be increasingly possible to sequence the entire genomes of additional individuals sampled from divergent populations and divergent species. Sampling this range of relatedness throughout the genus promises to greatly improve our understanding of genome evolution and the genetic basis and evolutionary breadth of the mechanisms underlying adaptation and evolution.

In conjunction with these technical advances, there is a clear need to establish the adaptive ecological significance of trait variation. Continued field experiments, including reciprocal transplants and ecological manipulations, will provide direct tests of putative selective mechanisms and evidence for local adaptation. With this combination of approaches available, Mimulus will continue to provide unique opportunities to expand and integrate our understanding of the interplay of ecology, evolution and genomics.

\section{Acknowledgements}

We thank the co-PIs on the FIBR grant, $\mathrm{T}$ Bradshaw, $\mathrm{F}$ Dietrich, L Fishman, D Schemske, J Tomkins, T Vision, members of their labs, and the international network of Mimulus researchers, for ongoing discussions about this research program. J Modliszewski provided helpful comments on the manuscript. Numerous collaborators at JGI have contributed to the development of Mimulus genomic resources. This material is based upon work supported by the National Science Foundation (FIBR Grant no. EF-0328636).

\section{References}

Allen WR, Sheppard PM (1971). Copper tolerance in some Californian populations of the monkey flower, Mimulus guttatus. Proc Roy Soc Lond Ser B 177: 177-196.

Angert AL, Schemske DW (2005). The evolution of species' distributions: reciprocal transplants across the elevation ranges of Mimulus cardinalis and M. lewisii. Evolution 59: 1671-1684.

Awadalla P, Ritland K (1997). Microsatellite variation and evolution in the Mimulus guttatus species complex with contrasting mating systems. Mol Biol Evol 14: 1023-1034.

Beardsley PM, Olmstead RG (2002). Redefining Phrymaceae: the placement of Mimulus, tribe Mimuleae, and Phryma. Am J Bot 89: 1093-1102.

Beardsley PM, Schoenig SE, Whittall JB, Olmstead RG (2004). Patterns of evolution in Western North American Mimulus (Phrymaceae). Am J Bot 91: 474-489.

Beardsley PM, Yen A, Olmstead RG (2003). AFLP phylogeny of Mimulus section Erythranthe and the evolution of hummingbird pollination. Evolution 57: 1397-1410.

Bleiweiss R (2001). Mimicry on the QT(L): genetics of speciation in Mimulus. Evolution 55: 1706-1709.

Bradshaw HD, Otto KG, Frewen BE, McKay JK, Schemske DW (1998). Quantitative trait loci affecting differences in floral morphology between two species of monkeyflower (Mimulus). Genetics 149: 367-382.

Bradshaw HD, Schemske DW (2003). Allele substitution at a flower colour locus produces a pollinator shift in monkeyflowers. Nature 426: 176-178.

Bradshaw HD, Wilbert SM, Otto KG, Schemske DW (1995). Genetic mapping of floral traits associated with reproductive isolation in monkeyflowers (Mimulus). Nature 376: 762-765.

Buckler ES, Phelps-Durr TL, Buckler CSK, Dawe RK, Doebley JF, Holtsford TP (1999). Meiotic drive of chromosomal knobs reshaped the maize genome. Genetics 153: 415-426.

Campbell DR, Waser NM (2001). Genotype-by-environment interaction and the fitness of plant hybrids in the wild. Evolution 55: 669-676. 
Christie P, MacNair MR (1984). Complementary lethal factors in two North American populations of the yellow monkeyflower. J Hered 75: 510-511.

Christie P, MacNair MR (1987). The distribution of postmating reproductive isolating genes in populations of the yellow monkeyflower, Mimulus guttatus. Evolution 41: 571-578.

Clausen J (1951). Stages in the Evolution of Plant Species. Hafner Publishing Co.: New York.

Colosimo PF, Hosemann KE, Balabhadra S, Villarrea G, Dickson M, Grimwood J et al. (2005). Widespread parallel evolution in sticklebacks by repeated fixation of ectodysplasin alleles. Science 5717: 1928-1933.

Coyne JA, Orr HA (2004). Speciation. Sinauer Associates: Sunderland, MA

Darwin C (1859). On the Origin of Species by Means of Natural Selection or the Preservation of Favored Races in the Struggle for Life. J. Murray: London.

Diaz A, MacNair MR (1999). Pollen tube competition as a mechanism of prezygotic reproductive isolation between Mimulus nasutus and its presumed progenitor M. guttatus. New Phytol 144: 471-478.

Dudash MR, Carr DE (1998). Genetics underlying inbreeding depression in Mimulus with contrasting mating systems. Nature 393: 682-684.

Feder ME, Mitchell-Olds T (2003). Evolutionary and ecological functional genomics. Nat Rev Genet 4: 649-655.

Fenster CB, Diggle PK, Barrett SCH, Ritland K (1995). The genetics of floral development differentiating two species of Mimulus (Scrophulariaceae). Heredity 74: 258-266.

Fenster CB, Ritland K (1994). The quantitative genetics of mating system divergence in the yellow monkeyflower species complex. Heredity 73: 422-435.

Fishman L, Kelly AJ, Morgan E, Willis JH (2001). A genetic map in the Mimulus guttatus species complex reveals transmission ratio distortion due to heterospecific interactions. Genetics 159: 1701-1716.

Fishman L, Kelly AJ, Willis JH (2002). Minor quantitative trait loci underlie floral traits associated with mating system divergence in Mimulus. Evolution 56: 2138-2155.

Fishman L, Willis JH (2001). Evidence for Dobzhansky-Muller incompatibilities contributing to the sterility of hybrids between Mimulus guttatus and M. nasutus. Evolution 55: 1932-1942.

Fishman L, Willis JH (2005). A novel meiotic drive locus almost completely distorts segregation in Mimulus (monkeyflower) hybrids. Genetics 169: 347-353.

Fishman L, Willis JH (2006). A cytonuclear incompatibility causes anther sterility in Mimulus hybrids. Evolution 60: 1372-1381.

Gardner M, Macnair M (2000). Factors affecting the co-existence of the serpentine endemic Mimulus nudatus Curran and its presumed progenitor, Mimulus guttatus Fischer ex DC. Biol J Linn Soc 69: 443-459.

Grant AL (1924). A monograph of the genus Mimulus. Ann Missouri Bot Gard 11: 99-388.

Grant KA, Grant V (1968). Hummingbirds and their flowers. J Ecol 58: 318-319.

Hall MC, Basten CJ, Willis JH (2006). Pleiotropic quantitative trait loci contribute to population divergence in traits associated with life-history variation in Mimulus guttatus. Genetics 172: 1829-1844.

Hall MC, Willis JH (2005). Transmission ratio distortion in intraspecific hybrids of Mimulus guttatus: implications for genomic divergence. Genetics 170: 375-386.

Hall MC, Willis JH (2006). Divergent selection on flowering time contributes to local adaptation in Mimulus guttatus populations. Evolution 60: 2466-2477.

Henikoff S, Ahmad K, Malik HS (2001). Speciation and centromere evolution. Science 294: 2478-2480.

Henikoff S, Malik HS (2002). Selfish drivers. Nature 417: 227.
Hiesey WM, Nobs MA, Bjorkman O (1971). Experimental Studies on the Nature of Species. V. Biosystematics, Genetics, and Physiological Ecology of the Erythranthe Section of Mimulus. Carnegie Institution of Washington: Washinton, DC.

Hughes R, Bachmann K, Smirnoff N, Macnair M (2001). The role of drought tolerance in serpentine tolerance in the Mimulus guttatus Fischer ex DC complex. S Afr J Sci 97: 581-586.

Jaillon O, Aury JM, Brunet F, Petit JL, Stange-Thomann N, Mauceli E et al. (2004). Genome duplication in the teleost fish Tetraodon nigroviridis reveals the early vertebrate protokaryotype. Nature 431: 946-957.

Kelly AJ, Willis JH (1998). Polymorphic microsatellite loci in Mimulus guttatus and related species. Mol Ecol 7: 769-774.

Kelly JK (2003). Deleterious mutations and the genetic variance of male fitness components in Mimulus guttatus. Genetics 164: 1071-1085.

Kelly JK, Willis JH (2001). Deleterious mutations and genetic variation for flower size in Mimulus guttatus. Evolution 55 937-942.

Kiang YT, Hamrick JL (1978). Reproductive isolation in the Mimulus guttatus - M. nasutus complex. Am Midl Nat $\mathbf{1 0 0}$ 269-276.

Leclerc-Potvin C, Ritland K (1994). Modes of self-fertilization in Mimulus guttatus (Scrophulariaceae): a field experiment. Am I Bot 81: 197-205.

Lin J-Z, Ritland K (1996). Construction of a genetic linkage map in the wild plant Mimulus using RAPD and isozyme markers. Genome 39: 63-70.

Lin J-Z, Ritland K (1997). Quantitative trait loci differentiating the outbreeding Mimulus guttatus from the inbreeding $M$. platycalyx. Genetics 146: 1115-1121.

Macnair M (1983). The genetic control of copper tolerance in the yellow monkeyflower, Mimulus guttatus. Heredity $\mathbf{5 0}$ 283-293.

Macnair M (1989). A new species of Mimulus endemic to copper mines in California. Bot I Linn Soc 100: 1-14.

Macnair M, Christie P (1987). The distribution of postmating reproductive isolating genes in populations of the yellow monkeyflower, Mimulus guttatus. Evolution 41: 571-578.

Macnair M, Cumbes QJ (1989). The genetic architecture of interspecific variation in Mimulus. Genetics 122: 211-222.

Macnair MR, Christie P (1983). Reproductive isolation as a pleiotropic effect of copper tolerance in Mimulus guttatus? Heredity 50: 295-302.

Macnair MR, Macnair VE, Martin BE (1989). Adaptive speciation in Mimulus: an ecological comparison of $M$. cupriphilis with its presumed progenitor, M. guttatus. New Phytol 112: 269-279.

Malik HS (2005). Mimulus finds centromeres in the driver's seat. Trends Ecol Evol 20: 151-154.

Martin NH, Willis JH (2007). Ecological divergence associated with mating system causes nearly complete reproductive isolation between sympatric Mimulus species. Evolution 61: 68-82.

McArthur ED, Alum HT, Eldredge FA, Tai W, Vickery RK (1972). Chromosome counts in section Simiolus of the genus Mimulus (Scrophulariaceae). IX. Polyploid and aneuploid patterns of evolution. Madrono 21: 417-420.

Ramsey J, Bradshaw HD, Schemske DW (2003). Components of reproductive isolation between the Monkeyflowers Mimulus lewisii and M. cardinalis (Phrymaceae). Evolution 57: 1520-1534.

Ramsey J, Schemske DW (2002). Neopolyploidy in flowering plants. Annu Rev Ecol Syst 33: 589-639.

Ritland CE, Ritland K (1989). Variation of sex allocation among eight taxa of the Mimulus guttatus species complex (Scrophulariaceae). Am J Bot 76: 1731-1739.

Robertson AW, Diaz A, MacNair MR (1994). The quantitative genetics of floral characters in Mimulus guttatus. Heredity $\mathbf{7 2}$ 300-311. 
Scannell DR, Byrne KP, Gordon JL, Wong S, Wolfe KH (2006) Multiple rounds of speciation associated with reciprocal gene loss in polyploid yeast. Nature 440: 341-345.

Schemske DW, Bradshaw HD (1999). Pollinator preference and the evolution of floral traits in monkeyflowers (Mimulus). Proc Natl Acad Sci USA 96: 11910-11915.

Soltis DE, Soltis PS, Tate JA (2003). Advances in the study of polyploidy since Plant Speciation. New Phytol 161: 173-191.

Stebbins GL (1950). Variation and Evolution in Plants. Columbia University Press: New York.

Stebbins GL (1970). Adaptive radiation of reproductive characters in angiosperms I: pollination mechanisms. Annu Rev Ecol Syst 1: 307-326.

Sweigart AL, Fishman L, Willis JH (2006). A simple genetic incompatibility causes hybrid male sterility in Mimulus. Genetics 172: 2465-2479.

Sweigart AL, Willis JH (2003). Patterns of nucleotide diversity in two species of Mimulus are affected by mating system and asymmetric introgression. Evolution 57: 2490-2506.

Turesson G (1922). The genotypical response of plant species to the habitat. Hereditas 3: 211-350.
Vickery RK (1978). Case studies in the evolution of species complexes in Mimulus. Evol Biol 11: 405-507.

Vickery RK (1995). Speciation by aneuploidy and polyploidy in Mimulus (Scrophulariaceae). Great Basin Nat 55: 174-176.

Waser NM, Vickery RK, Price MV (1982). Patterns of seed dispersal and population differentiation in Mimulus guttatus. Evolution 36: 753-761.

Werth SR, Windham MD (2001). A model for divergent, allopatric speciation of polyploid pteridophytes resulting from silencing of duplicate-gene expression. Am Nat 137: 515-526.

Whittall JB, Carlson ML, Beardsley PM, Meinke RJ, Liston A (2006). The Mimulus moschatus alliance (Phrymaceae): molecular and morphological phylogenetics and their conservation implications. Syst Bot 31: 380-397.

Wolfe KH, Shields DC (1997). Molecular evidence for an ancient duplication of the entire yeast genome. Nature 387 : 708-713.

Zeh JA, Zeh DW (2005). Maternal inheritance, sexual conflict, and the maladapted male. Trends Genet 21: 281-286. 